\title{
Grid Drawings and the Chromatic Number
}

\author{
Martin Balko \\ Department of Applied Mathematics, \\ Charles University, Faculty of Mathematics and Physics, \\ Malostranské nám. 25, 11800 Praha 1, Czech Republic \\ balko@kam.mff.cuni.cz
}

\begin{abstract}
A grid drawing of a graph maps vertices to the grid $\mathbb{Z}^{d}$ and edges to line segments that avoid grid points representing other vertices. We show that a graph $G$ is $q^{d}$-colorable, $d, q \geq 2$, if and only if there is a grid drawing of $G$ in $\mathbb{Z}^{d}$ in which no line segment intersects more than $q$ grid points. This strengthens the result of D. Flores Pen̋aloza and F. J. Zaragoza Martinez. Second, we study grid drawings with a bounded number of columns, introducing some new NP-complete problems. Finally, we show that any planar graph has a planar grid drawing where every line segment contains exactly two grid points. This result proves conjectures asked by D. Flores Pen̋aloza and F. J. Zaragoza Martinez.
\end{abstract}

Keywords: graph drawings, grid, graph coloring, chromatic number.

\section{Introduction}

Let $G=(V, E)$ be a simple, undirected and finite graph. A $k$-coloring of $G$ is a function $f: V \rightarrow C$ for some set $C$ of $k$ colors such that $f(u) \neq f(v)$ for every edge $u v \in E$. If such $k$-coloring of $G$ exists, then $G$ is $k$-colorable. The chromatic number $\chi(G)$ of $G$ is the least $k$ such that $G$ is $k$-colorable.

Let $\overline{x y}$ denote the closed straight line segment joining two grid points $x, y \in$ $\mathbb{Z}^{d}$. The line segment $\overline{x y}$ is primitive if $\overline{x y} \cap \mathbb{Z}^{d}=\{x, y\}$.

Definition 1. A grid drawing $\phi(G)$ of $G$ in $\mathbb{Z}^{d}$ is an injective mapping $\phi: V \rightarrow$ $\mathbb{Z}^{d}$ such that, for every edge uv $\in E$ and vertex $w \in V, \phi(w) \in \overline{\phi(u) \phi(v)}$ implies that $w=u$ or $w=v$.

That is, a grid drawing represents vertices of $G$ by distinct grid points in $\mathbb{Z}^{d}$, and each edge by a straight line segment between its endpoints such that the only vertices an edge intersects are its own endpoints. Note that we allow edges to cross in a grid drawing.

\footnotetext{
* A four-page abstract of an earlier version of this paper appeared in the proceedings of the 28th European Workshop on Computational Geometry EuroCG '12 [1]. The booklet of abstracts is available here: http://www.diei.unipg.it/eurocg2012/ booklet.pdf
} 

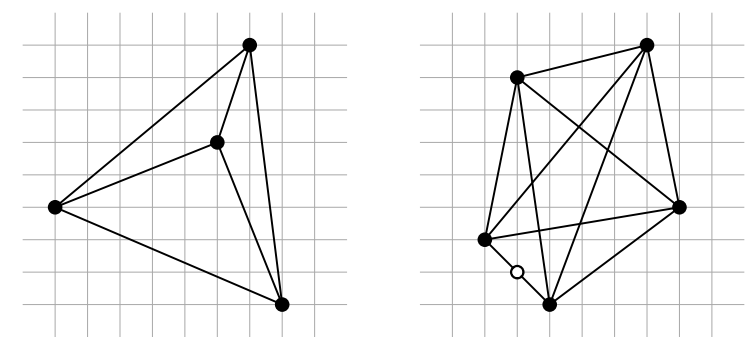

Fig. 1. Examples of grid drawings

\section{Grid Drawings}

In this paper we study grid drawings from three points of view - robustness, compactness and planarity.

\subsection{Robustness}

The robustness of a grid drawing is understood as the maximum number of grid points a line segment of such a drawing contains. For a given graph $G$ we would like to find a grid drawing of $G$ with the lowest robustness.

A graph $G$ is said to be (grid) locatable in $\mathbb{Z}^{d}$ if there exists a grid drawing of $G$ in $\mathbb{Z}^{d}$ where every edge is represented by a primitive line segment (such a drawing is also called primitive). Finding a primitive grid drawing of $G$ is called locating the graph G. D. Flores Pen̈aloza and F. J. Zaragoza Martinez [5] showed the following characterization:

Theorem 1 ([5]). A graph $G$ is locatable in $\mathbb{Z}^{2}$ if and only if $G$ is 4 -colorable.

Therefore not all graphs are locatable in $\mathbb{Z}^{2}$ and every (two-dimensional) grid drawing of any $k$-colorable graph, $k>4$, contains a line segment which intersects at least three grid points. This leads us to a generalization of the concept of locatability.

Definition 2. A graph $G$ is (grid) $q$-locatable in $\mathbb{Z}^{d}$, for some integer $q \geq 2$, if there exists a grid drawing $\phi(G)$ in $\mathbb{Z}^{d}$ in which every line segment intersects at most $q$ grid points.

For example the grid drawing in Figure 1 shows that $K_{5}$ is three-locatable in $\mathbb{Z}^{2}$ (the third grid point on one line segment is denoted by an empty circle). Using this new definition we can prove a much stronger version of Theorem 1 .

Theorem 2. For integers $d, q \geq 2$, a graph $G$ is $q^{d}$-colorable if and only if $G$ is q-locatable in $\mathbb{Z}^{d}$.

The proof is constructive (see Section 3) and for a given coloring of $G$ with at most $q^{d}$ colors we get a linear time algorithm which gives us a grid drawing $\phi(G)$ of $G$ in $\mathbb{Z}^{d}$ where every line segment contains at most $q$ grid points. 
Corollary 1. A graph is $2^{d}$-colorable, $d \geq 2$, if and only if it is locatable in $\mathbb{Z}^{d}$.

Corollary 2. For given integers $d, q \geq 2$ and a graph $G$, it is NP-complete to decide whether or not $G$ is q-locatable in $\mathbb{Z}^{d}$.

Proof. Theorem 2 shows a reduction of the Colorability Problem, which asks "Does $G$ admit a proper vertex coloring with $q^{d}$ colors?", to our problem.

\subsection{Compactness}

The compactness of a grid drawing signifies bounded space of the drawing in some fashion. Our aim here is to draw the graph in the smallest number of columns. For an integer $d \geq 2$, a column in the grid $\mathbb{Z}^{d}$ with $\operatorname{rank}\left(x_{1}, \ldots, x_{d-1}\right) \in$ $\mathbb{Z}^{d-1}$ is the set $\left\{\left(x_{1}, \ldots, x_{d-1}, x\right) \mid x \in \mathbb{Z}\right\}$. It turns out this problem is closely related to a special form of a defective coloring in which some colors induce independent sets and some disjoint unions of paths (linear forests). We call these colorings mixed. Other measure of the compactness of a grid drawing is its volume. This type of compactness has been studied by D. R. Wood and A. Pór [10].

Definition 3. If there is a mixed coloring of a graph $G$ such that a colors induce independent sets (so called normal colors) and $b$ colors induce linear forests (path colors), then we say that $G$ is $(a, b)$-colorable.

For nonprimitive grid drawings, there is no loss of generality in assuming that the grid is two-dimensional, as we can transfer a grid drawing $\phi(G)$ in $\mathbb{Z}^{d}, d>2$, on $l$ columns, to $l$ columns in $\mathbb{Z}^{2}$ (we might have to shift some columns higher so that no point representing a vertex lies on a non-incident line segment). By the same trick, we can also assume that there is no unused column between two columns in our drawing. If $l$ is the minimum number of columns on which $G$ can be drawn, then we say that such grid drawing of $G$ is compact. Theorem 2 gives us the following upper bound:

Corollary 3. Every compact grid drawing of a graph $G$ has at most $\chi(G)$ columns.

The following simple proposition characterizes which graphs are drawable on $l$ columns in the terms of the graph theory.

Proposition 1. A graph $G$ is drawable on $l$ columns if and only if $G$ is $(0, l)$ colorable.

Proof. We denote the path color classes as $V_{1}, V_{2}, \ldots, V_{l}$. In the grid drawing of $G$ on $l$ columns, the vertices represented by points of a single column define the set $V_{i}$. On the other hand, each induced subgraph $G\left[V_{i}\right]$ can be drawn on a single column and we can always shift the vertices in such way that the visibility of representing points is guaranteed. 
If we restrict our attention to only primitive grid drawings, then the situation changes radically. According to Theorem 2, we have to proceed to grid drawings in higher dimensions as the chromatic number grows. Despite this, the following theorem gives us a characterization of locating similar to Proposition 1.

Theorem 3. Let $G$ be a graph and let $d, l \geq 2$ be integers such that $2^{d-1}<l \leq$ $2^{d}$. Then $G$ can be located on l columns in $\mathbb{Z}^{d}$ if and only if $G$ is $\left(2 l-2^{d}, 2^{d}-l\right)$ colorable.

The second part says that $G$ can be colored with at most $l$ colors and at most $2^{d}-l$ of them are path colors. Note that if $l>2^{d}$, then $G$ cannot be located in $\mathbb{Z}^{d}$ at all, because, according to Corollary 1, only $2^{d}$-colorable graphs can. Also note that the dimension of grid in the statement of Theorem 3 is minimum for such choice of $l$, according to Corollary 1 . The proof (see Section 4) of this theorem also shows how to relocate a primitive grid drawing of $G$ on a bounded number of columns, such that the new grid drawing is still primitive and it also requires a small part of the grid. We can also describe the case for $l \leq 2^{d-1}$ and depict the relation between nonprimitive and primitive grid drawings.

Corollary 4. Let $G$ be a graph with a grid drawing on l columns. Then $G$ can be located in $\mathbb{Z}^{\left\lceil\log _{2} k\right\rceil}$ on $k$ columns, where $k$ is some number in the range $l \leq k \leq 2 l$.

Proof. The lower bound on $k$ is apparent. For the upper bound, we just combine Proposition 1 and Theorem 3 and we split every path color into two normal ones.

Proposition 2. Let $G$ be a graph and let $d \geq 2, l$ be integers such that $1 \leq l \leq$ $2^{d-1}$ Then $G$ can be located on $l$ columns in $\mathbb{Z}^{d}$ if and only if $G$ is drawable on $l$ columns (in $\left.\mathbb{Z}^{2}\right)$.

Proof. The first implication is apparent. To prove the reverse one, it suffices to take each column of the drawing in $\mathbb{Z}^{2}$ and copy it on a unique column with rank from the set $\left\{\left(r_{1}, \ldots, r_{d-1}\right) \mid r_{i} \in\{0,1\}\right\} \subset \mathbb{Z}^{d-1}$.

These characterizations by mixed colorings seem to be useful, as we can use them to prove various results - for example the following theorem (see Section 5):

Theorem 4. Let $a$ and $b$ be given nonnegative integers such that $a+b \geq 2$ and $(a, b) \neq(2,0)$. Then it is NP-complete to decide whether or not a graph $G=(V, E)$ is $(a, b)$-colorable.

As a consequence we obtain that drawing/locating of graphs on a bounded number of columns is a difficult task answering the open question in [2]. This problem was already partially solved, as Chappell, Gimbel and Hartman [3] proved that determining whether $G$ can be colored with at least two path colors is NPcomplete. However this does not answer the question for locating of graphs.

Corollary 5. For a given integer $l \geq 2$ and a graph $G$, it is NP-complete to decide whether or not it is possible to draw a given graph on l columns. 
Corollary 6. For a given $l \geq 2$ and $G$, it is NP-complete to decide whether or not it is possible to locate a given graph on l columns (in $\mathbb{Z}^{\left\lceil\log _{2} l\right\rceil}$ ).

Using mixed colorings we can show some other results. For example, as every planar graph is (0,3)-colorable (see [7]), we get the following:

Proposition 3. Every planar graph can be drawn on three columns.

Cáceres et. al. 2] showed that every outerplanar graph can be drawn (and located) on two columns. In the same paper there is an example of a planar graph which is not $(2,1)$-colorable. Thus we need four columns to locate an arbitrary planar graph.

Although locating of graphs on a bounded number of columns is NP-complete, we can show some estimates on the number of columns which depend on the maximum degree of a graph.

Theorem 5. Let $G=(V, E)$ be a graph with $\Delta(G) \leq 2^{d+1}-1$, for $d \in \mathbb{N}$. Then $G$ can be located on $2^{d}$ columns in $\mathbb{Z}^{d+1}$.

Lemma 1 ([9]). Let $G=(V, E)$ be a graph and let $k_{1}, k_{2}, \ldots, k_{m}$ be nonnegative integers with $k_{1}+k_{2}+\ldots+k_{m} \geq \Delta(G)-m+1$. Then $V$ can be partitioned into $V_{1}, V_{2}, \ldots, V_{m}$ so that $\Delta\left(G\left[V_{i}\right]\right) \leq k_{i}$, for all $i \in[m]$.

Proof (of Theorem 5). According to Proposition 1 and 2 it suffices to show by induction on $d$ that the assumption in the theorem implies that $G$ is $\left(0,2^{d}\right)$ colorable. For $d=1$ we can use the proof of a theorem proved in [2] which says that every graph with maximum degree at most three can be located on two columns in $\mathbb{Z}^{2}$.

For the inductive step - let the maximum degree of $G$ be at most $2^{d+1}-1$. Then, according to Lemma 1, $V$ can be partitioned into $V_{1}$ and $V_{2}$ such that $\Delta\left(G\left[V_{1}\right]\right) \leq 2^{d}-1$ and $\Delta\left(G\left[V_{2}\right]\right) \leq 2^{d}-1$, if we set $m=2$ and $k_{1}=k_{2}=2^{d}-1$. It follows from the inductive step that the vertices of each of the graphs $G\left[V_{1}\right]$, $G\left[V_{2}\right]$ can be partitioned into $2^{d-1}$ required sets. Together these partitions give the partition of $V$ into $2^{d-1}+2^{d-1}=2^{d}$ sets.

This bound is sharp in certain cases, for example for $K_{2^{d+1}-1}$, as the minimum number of columns on which we can draw $K_{n}$ is $\left\lceil\frac{n}{2}\right\rceil$.

\subsection{Planarity}

Although Theorem 2 and the Four Color Theorem imply that every planar graph is locatable in $\mathbb{Z}^{2}$, the drawings obtained by this approach do not have to be planar. On the other hand, De Fraysseix, Pach, and Pollack [6], Schnyder [11, and Chrobak and Nakano [4 proved that any planar graph on $n$ vertices has a planar grid drawing which can be realized in grid of size $O(n) \times O(n)$. Unfortunately, these drawings are not necessarily primitive. We show that there is a primitive and planar grid drawing of any planar graph (such grid drawings are called proper). This settles the conjecture of D. Flores Peñaloza and F. J. Zaragoza Martinez [5]. 
Theorem 6. There exists a proper grid drawing for every planar graph $G$.

To prove this statement we use the Four Color Theorem (see Section 6) for a sketch of a proof. These two results are actually equivalent, as the other implication is apparent if we consider the function $g: \mathbb{Z}^{2} \rightarrow \mathbb{Z}_{2}^{2}$,

$$
g(x, y)=(x(\bmod 2), y(\bmod 2))
$$

as the coloring of $G$. The choice of coordinates in the proof also gives us a coloring of $G$ with at most four colors. In fact, we also prove a stronger conjecture from [5].

Corollary 7. Any planar graph $G$ is isomorphic to a plane subgraph $H$ of the visibility graph of the integer lattice, in such a way that the function $g$ is a coloring of $H$ that uses exactly $\chi(G)$ colors.

The rough estimation of the size of the obtained grid drawings is $O\left(n^{2}\right) \times$ $O\left(n^{2 \Delta+2}\right)$, where $\Delta$ is the maximum degree of a given graph. This is an exponential bound for graphs with linear maximum degree and unfortunately we do not know if it can be reduced for every planar graph. Thus we state the following conjecture.

Conjecture 1. For an arbitrary planar graph $G$, is there a proper grid drawing of $G$ in a grid of polynomial size?

\section{Proof of Theorem 2}

Lemma 2. For $d, q \geq 2$, if a graph is $q$-locatable in $\mathbb{Z}^{d}$, then it is $q^{d}$-colorable.

Proof. A trivial but useful observation is that the line segment $\overline{a b}$ between the grid points $a, b \in \mathbb{Z}^{d}$ intersects exactly the grid points of the form

$$
\left(a_{1}+i \frac{b_{1}-a_{1}}{\alpha}, \ldots, a_{d}+i \frac{b_{d}-a_{d}}{\alpha}\right)
$$

where $0 \leq i \leq \alpha$ and $\alpha=\operatorname{gcd}\left(\left|a_{1}-b_{1}\right|, \ldots,\left|a_{d}-b_{d}\right|\right)$. Let $\phi(G)$ be a grid drawing of the graph $G=(V, E)$ in $\mathbb{Z}^{d}$ having at most $q$ grid points on line segment. Consider the function $f: \mathbb{Z}^{d} \rightarrow \mathbb{Z}_{q}^{d}$ denoted as

$$
f\left(x_{1}, \ldots, x_{d}\right)=\left(x_{1}(\bmod q), \ldots, x_{d}(\bmod q)\right) .
$$

We use $f$ as a coloring of the grid with $q^{d}$ colors and we show that it is also a proper vertex coloring of $G$. Assume to the contrary that $f(\phi(u))=f(\phi(v))$ for some $u v \in E$ where $\phi(u)=\left(u_{1}, \ldots, u_{d}\right)$. Then $u_{1} \equiv v_{1}, \ldots, u_{d} \equiv v_{d}(\bmod q)$ which implies

$$
\operatorname{gcd}\left(\left|u_{1}-v_{1}\right|, \ldots,\left|u_{d}-v_{d}\right|\right) \geq q .
$$

According to our observation, there are at least $q+1$ grid points lying on the line segment $\overline{\phi(u) \phi(v)}$. This contradicts the fact that $G$ is $q$-locatable via the drawing $\phi(G)$. 
This proves the first part of Theorem 2 and thus it remains to show the implication in the opposite direction. The main idea is to find a subset of $\mathbb{Z}^{d}$ which we can use for a convenient grid drawing of every $q^{d}$-colorable graph.

Assume that the dimension $d$ is fixed and let $p$ be a prime number. We define $V_{p, 1}$ as the sequence $\left\{x_{i}\right\}_{i=0}^{p^{d}-1}$ such that each $x_{i}$ is from the set $\mathbb{Z}_{p}^{d}$ and no two terms are equal. This definition is correct, as we can always find $p$ distinct residues modulo $p$ and, naturally, there are $p^{d}$ distinct $d$-tuples of these residues. Now we define $V_{p, e}$ for $e \geq 2$ inductively. Assume as induction hypothesis that we have already set $V_{p, e-1}$. Now we place $V_{p, e}$ as a chain of $p^{d}$ copies of $V_{p, e-1}$. Then we change the terms on the positions $i+p^{d(e-1)}, \ldots, i+\left(p^{d}-1\right) p^{d(e-1)}$ for every $i \in\left\{0,1, \ldots, p^{d(e-1)}-1\right\}$ in such way that the new terms are numbers from $\mathbb{Z}_{p^{e}}$ congruent to their predecessors modulo $p^{e}$ and no two terms in $V_{p, e}$ are equal. For each element of $Z_{p^{e-1}}^{d}$ there are $p^{d}$ congruent elements from $\mathbb{Z}_{p^{e}}^{d}$ modulo $p^{e-1}$ and one of them is on the $i$-th position of $V_{p, e}$. Thus the definition of $V_{p, e}$ is, again, correct.

Continual repeating of the copies of $V_{p, e}$ gives us the infinite sequence $S_{p, e}$. We denote the $i$-th term of $S_{p, e}$ as $S_{p, e}[i]$ and the distance of two terms $S_{p, e}[i]$ and $S_{p, e}[j]$ is given by $|i-j|$. The following lemma shows an important feature of these sequences.

Lemma 3. Let $p$ be prime number and e a positive integer. Then two terms of $S_{p, e}$ are equal if and only if $p^{\text {de }}$ divides their distance.

Proof. Two distinct terms on positions $i \neq j$ are equal if and only if they are both in different copies of $V_{p, e}$, but on the same position in $V_{p, e}$. The length of $V_{p, e}$ is exactly $p^{d e}$, so the distance between $S_{p, e}[i]$ and $S_{p, e}[j]$ is a multiple of $p^{d e}$.

Given an integer $s$, we set $f(p)$ as $\min \left\{e \in \mathbb{N} \mid p^{d e} \geq s\right\}$ for every prime number $p<s$. Now, for every $i$, where $0 \leq i \leq s-1$, we choose a distinct column of $\mathbb{Z}^{d}$ such that for every prime number $p<s$ the rank of this column is congruent to the first $d-1$ elements of the $d$-tuple $S_{p, e}[i]$ modulo $p^{f(p)}$. We label the chosen columns as $W_{0, s}, W_{1, s}, \ldots, W_{s-1, s}$. In every column $W_{i, s}$ we keep only the points with their last coordinate congruent to the last element of $S_{p, e}[i]$ modulo $p^{f(p)}$, again for every $p<s$. Finally we set $W_{s}=\bigcup_{i=0}^{s-1} W_{i, s}$.

If there is a prime $p \geq s$ such that ranks of two or more columns from $W_{s}$ are congruent modulo $p$, then we assign distinct residues modulo $p$ to these columns. Subsequently, we keep only the points with their last coordinate congruent to the assigned residue modulo $p$ in each one of these columns. This method is correct, because the number of possible residues is at least $s$, thus every column can get a unique residue. According to the Chinese Remainder Theorem, every column of $W_{s}$ still contains infinitely many points. The construction of the set $W_{s}$ is not easy to describe, but it has nice properties that allow us to prove the crucial lemma in the proof of Theorem 2 . 
Lemma 4. Let $s$ be $d$-th power of integer $q \geq 2$. Let $a=\left(a_{1}, \ldots, a_{d}\right), b=$ $\left(b_{1}, \ldots, b_{d}\right)$ be grid points located in distinct columns of the set $W_{s}$. Then

$$
\operatorname{gcd}\left(\left|a_{1}-b_{1}\right|, \ldots,\left|a_{d}-b_{d}\right|\right) \leq q-1 .
$$

Proof. Let $\alpha$ denote the greatest common divisor in the statement. Assume that the grid point $a$ is in the column $W_{x, s}$ and the grid point $b$ in the column $W_{y, s}$, $0 \leq x, y \leq s-1$ and $x \neq y$. The last remark in the construction of $W_{s}$ guarantees that no prime number larger than $s-1$ divides $\alpha$. Also, for every $e \in \mathbb{N}$ and prime number $p$, the power $p^{e}$ divides $\alpha$ if and only if $S_{p, e}[x]=S_{p, e}[y]$. Since $p^{e} \mid \alpha$ implies that each coordinate of $a$ is congruent to each coordinate of $b$ modulo $p^{e}$ and these coordinates are congruent to the $d$-tuples $S_{p, e}[x]$ and $S_{p, e}[y]$ modulo $p^{e}$. Thus $p^{e}$ does not divide $\alpha$ for $e \geq f(p)$. Otherwise $S_{p, f(p)}[x]=S_{p, f(p)}[y]$ and, according to Lemma 3, the distance $|x-y|$ between them is at least $p^{d f(p)}$, which is at least $s$. But this contradicts the inequality $0 \leq x, y \leq s-1$.

So we can assume that $\alpha=\prod_{i=1}^{k} p_{i}^{e_{i}}$ where $p_{i}$ are prime numbers and $1 \leq$ $e_{i} \leq f\left(p_{i}\right)-1$. Then

$$
\alpha^{d}=\prod_{i=1}^{k} p_{i}^{d e_{i}} \leq s-1
$$

holds. Since the expression of $\alpha$ implies that $S_{p_{i}, e_{i}}[x]=S_{p_{i}, e_{i}}[y]$ and, again, we get $p_{i}^{d e_{i}}|| x-y \mid$ for every $i \in\{1,2, \ldots, k\}$. Thus $\alpha^{d} \leq|x-y| \leq s-1=q^{d}-1$, which implies $\alpha \leq \sqrt[q]{q^{d}-1}$. Since $\alpha$ is an integer, $\alpha \leq q-1$.

Now we can finally prove Theorem 2, The first implication is proved in Lemma 2 , so assume that $G$ is a $q^{d}$-colorable graph, $q \geq 2$. We need to find a grid drawing of $G$ such that at most $q$ grid points lie on any of its line segments. It suffices to show how to find such drawing for the complete $q^{d}$-partite graph $K\left(q^{d}, n\right)$ with $n \in \mathbb{N}$ vertices in each color class, as every $q^{d}$-colorable graph on $n$ vertices is its subgraph. We consider the set $W_{s}$ for $s=q^{d}$ and we keep only the first $n$ vertices of its first two columns. Then for every $i, 2 \leq i \leq s-1$, we keep the first $n$ points in the column $W_{i, s}$ such that all points in previous columns are visible from any of these points (with respect to other columns). These points in $W_{i, s}$ exist, because, unlike $W_{i, s}$, the previous columns are finite sets. Afterwards we obtain the set $W_{s}(n) \subset W_{s}$ such that $K\left(q^{d}, n\right)$ is isomorphic to the visibility graph $v\left(W_{s}(n)\right)$ and, according to Lemma 4, no line segment contains more then $q$ grid points. Therefore we get a suitable grid drawing of $G$ and the second implication is also proved.

\section{Proof of Theorem 3}

Suppose that $G$ is located on $l$ columns in $\mathbb{Z}^{d}$. We construct a congruence graph $C$ on the set of column ranks of such primitive grid drawing. Every vertex of this graph corresponds to a unique column rank and two vertices are adjacent if the corresponding ranks are congruent modulo two. The graph $C$ is a disjoint union of complete graphs and it contains at most $2^{d-1}<l$ isolated vertices, as 
the maximum number of possible values of ranks modulo two is $2^{d-1}$. All points in the columns with ranks which lie in the same connected component of $C$ can be colored with two normal colors. Since if we color the points with the odd last coordinate white and the rest black, then no two monochromatic points can share an edge, otherwise the line segment joining them would not be primitive.

Now we show by induction on $l$ that $l$ colors is enough and that there is always at most $2^{d}-l$ path colors among them. Consider the case when $l=2^{d-1}+1$. Then we color the points of every column, whose rank corresponds to an isolated vertex in $C$, with a single path color, obtaining at most $l-1$ path colors. Afterwards we color the points in all columns with ranks congruent modulo two using only two normal colors. Then the number of path colors decreases for every isolated vertex which becomes a larger component of $C$. This happens at least once, as $2^{d-1}<l$. Thus we have at most $l-2=2^{d}-l$ path colors. Note that the total number of colors is at most $l$, as we add at most one new color for every vertex. Thus the case for $l=2^{d-1}+1$ is done.

Let us assume that this initial congruence graph contains all isolated vertices of the final congruence graph. Now suppose that $C$ contains $l$ vertices. We know from the induction hypothesis that the condition holds for congruence graphs on $l-1$ vertices. We get the graph $C$ by joining one nonisolated vertex $u$ to such smaller congruence graph. If we join $u$ to some component of $C$ which is colored with two normal colors, then we color the points of a corresponding column with these colors. One color for points in even height, the other one for points in odd height. If $2^{d}-l$ drops bellow the number of used path colors, then we choose an isolated vertex whose column is colored with a single path color and color its points using two normal colors (one color is new for $u$ ).

If we join the new vertex $u$ to a component colored with a path color, then it is an isolated vertex $v$ and we can color points in the columns with ranks $u$ and $v$ using two normal colors. One is new for $u$, the other is original. The number of path colors is at most $2^{d}-l$ and we added at most one new normal color in each step. Thus the conditions on the number of path and normal colors holds.

Now we prove the reverse implication. Let $V_{1}, V_{2}, \ldots, V_{l}$ be the colors (both normal and path) of our mixed coloring. Consider the set

$$
\left\{\left(r_{1}, r_{2}, \ldots, r_{d-1}\right) \in \mathbb{Z}^{d-1} \mid r_{1} \in \mathbb{Z}_{4}, r_{i} \in \mathbb{Z}_{2}\right\} .
$$

The last $d-2$ coordinates $r_{2}, r_{3}, \ldots, r_{d-1}$ determine the set which we mark as $S_{r_{2}, \ldots, r_{d-1}}$ and its elements as $s_{i, r_{2}, \ldots, r_{d-1}}=\left(i, r_{2}, \ldots, r_{d-1}\right)$, for $i=0,1,2,3$. For $d=2$, there is only one such $S=\{0,1,2,3\}$. Now we repeat the following steps until there is no set of vertices left in our partition.

1. Take $S_{r_{2}, \ldots, r_{d-1}}$ that has not been chosen yet.

2. If there are two path colors $V_{i}, V_{j}$ and no normal color, then map $V_{i}$ to points of the column $s_{0, r_{2}, \ldots, r_{d-1}}$ and $V_{j}$ to points of the column $s_{1, r_{2}, \ldots, r_{d-1}}$.

3. If there is a path color $V_{i}$ and two normal colors $V_{j}, V_{k}$, then map $V_{i}$ to points of column $s_{1, r_{2}, \ldots, r_{d-1}}, V_{j}$ to points of column $s_{0, r_{2}, \ldots, r_{d-1}}$ with even $d$-th coordinate and $V_{k}$ to points of column $s_{2, r_{2}, \ldots, r_{d-1}}$ with odd $d$-th coordinate. 
4. If there is no path color, then take four (or two, if there are not that many) normal colors $V_{i}, V_{j}, V_{k}$ and $V_{m}$. Map $V_{i}$ to points of column $s_{0, r_{2}, \ldots, r_{d-1}}$ with even $d$-th coordinate divisible by three, $V_{j}$ to points of column $s_{1, r_{2}, \ldots, r_{d-1}}$ with even $d$-th coordinate, $V_{k}$ to points of column $s_{2, r_{2}, \ldots, r_{d-1}}$ with odd last coordinate and $V_{m}$ to points of column $s_{3, r_{2}, \ldots, r_{d-1}}$ with odd last coordinate not divisible by three.

5. Remove the chosen colors.

The maximum number of possible steps is $2^{d-2}$. This number is sufficient, as we can pair each path color $V_{i}$ with a unique empty color and we obtain $2^{d}$ colors in total and each step of the algorithm takes four of these colors and locates their vertices. Thus we can locate vertices of all these $2^{d}=4 \cdot 2^{d-2}$ colors within $2^{d-2}$ steps. It is not difficult to see that the obtained grid drawing is primitive. This completes the proof of Theorem 3 .

\section{Proof of Theorem 4}

Lemma 5. It is NP-complete to decide whether a given graph is $(1,1)$-colorable.

Proof. Let $F$ be a collection of $m$ clauses $C_{1}, C_{2}, \ldots, C_{m}$ over $n$ Boolean variables $v_{1}, v_{2}, \ldots, v_{n}$ such that each $C_{i}$ contains exactly three literals $c_{i, 1}, c_{i, 2}$ and $c_{i, 3}$. Each literal $c_{i, j}, i \in[m]$ and $j \in[3]$, is either $v_{k}$ or $\overline{v_{k}}$ for some suitable $k \in$ $[n]$. One-in-three 3SAT is a problem of determining whether there is a truth assignment satisfying $F$ such that each clause has exactly one true literal.

We construct a graph $G(k)$ shown in Figure 2 for each variable $v_{k}$. Then, for each $C_{i}$, we construct a graph $G\left(C_{i}\right)$ isomorphic to $K_{3}$ such that each of its vertices represents a different literal of $C_{i}$. Let $G(F)$ be a graph consisting of all the graphs $G(k)$ and $G\left(C_{i}\right)$ where the vertex $c_{i, j}$ is adjacent to $v \in V(G(k))$ if and only if the literal $c_{i, j}$ is $v \in\left\{v_{k}, \overline{v_{k}}\right\}$.

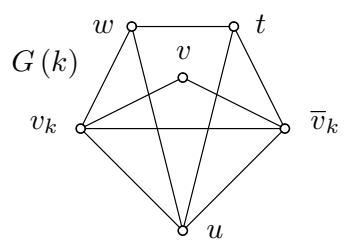

Fig. 2. The graph $G(k)$

Suppose that $G$ is colored with one path and one normal color, say black and white. Then the vertices $v_{k}$ and $\bar{v}_{k}$ of $G(k)$ are colored differently. Otherwise they are black and the vertex $u$ must be white. But then $w$ and $t$ are black and induce a black 4-cycle together with $v_{k}$ and $\bar{v}_{k}$. Also, if the vertices $x \in\left\{v_{k}, \bar{v}_{k}\right\}$ and $c_{i, j}$ are adjacent, then their colors are different too. Otherwise they are both black and we know that $\bar{v}_{k}$ is white and thus $u$ and $v$ are black. But then $v_{k}$ has three black neighbors which is a contradiction. We define the truth assignment 
$e$ for $F$ as follows: if $v_{k}$ is black, then $e\left(v_{k}\right)$ is true else $e\left(v_{k}\right)$ is false. The assignment $e$ is correct, as the vertices $v_{k}$ and $\bar{v}_{k}$ are not monochromatic. In addition, there is exactly one true literal in every clause. Otherwise there would be a black 3-cycle or an edge with both vertices white in some $G\left(C_{i}\right)$.

Suppose that $e$ satisfies $F$ such that every clause has exactly one true literal. Then we color the labeled vertices of each $G\left(C_{i}\right)$ white, if the corresponding literal is true; otherwise black. After that, we color the vertex $v \in\left\{v_{k}, \bar{v}_{k}\right\}$ adjacent to $c_{i, j}$ black (white, respectively) if $c_{i, j}$ is white (black, respectively). Note that the vertices $v_{k}$ and $\bar{v}_{k}$ are differently colored. It remains to color the rest of graph $G(k)$ for each $k \in[n]$.

Lemma 6 ([3]). It is NP-complete to decide whether a graph is $(0,2)$-colorable.

The known proof of this lemma is based on the result with so called one-defective colorings, but it is not difficult to modify the proof of Lemma 5 using the reduction of Not-All-Equal 3SAT. Now we can prove Theorem 4 by a reduction of the Colorability Problem. If we consider the number of colors $k=a+b$, then we can assume, according to Lemma 5 and 6 , that $k \geq 3$. Let $G$ be a given graph, then we create a graph $H$ by joining two disjoint copies of the complete graph $K_{a+2 b-1}$ to every vertex $v$ of $G$.

If $G$ is colored with $k$ normal colors, then we can color each clique with $a$ path and $b$ normal colors. On the other hand, if $H$ is colored with $a$ normal and $b$ path colors, then $G$ is colored with at most $k=a+b$ normal colors. If there is an edge $u v$ with both vertices colored with the same path color (say black) in $G$. Then $u$ has at least three black neighbors, but this is a contradiction.

\section{Sketch of the Proof of Theorem 6}

Proof (sketch). Let $G=(V, E)$ be a planar graph and let $\phi(G)$ be its initial planar embedding which maps vertices of $G$ to points of $\mathbb{R}^{2}$ and edges to line segments (its existence is ensured by Fáry's theorem). Let $f: V \rightarrow C$ be a vertex coloring of $G$ with four colors $(0,0),(0,1),(1,0)$ and $(1,1)$ ( $f$ exists according to the Four Color Theorem). Let $r \in \mathbb{R}^{+}$be the largest distance such that every vertex can be shifted by $r$ in any direction so that the condition on planarity still holds. Thus, for every $v \in V$, there is an open neighborhood $\Omega(v, r)$ of $\phi(v)$ such that any $x \in \Omega(v, r)$ can represent the vertex $v$ without violating planarity. We also choose $r$ small enough such that the neighborhoods of vertices which do not lie on the same vertical line are not crossed by the same vertical line.

Now we put vertical lines across $\mathbb{R}^{2}$ such that the distance between two consecutive lines is $\epsilon>0$ and every neighborhood is crossed by at least six lines. Then we choose one line and declare it as the initial line. Each line gets a number according to its order, the initial line has number zero. Now for every $v \in V$, we set $\phi(v)=x$, where $x \in \Omega(v, r)$ lies on some vertical line with number $l \equiv f(v)_{1}(\bmod 2), l \equiv f(v)_{1}(\bmod 3)$. We can always choose such line, because there are six consecutive lines crossing $\Omega(v, r)$ and their numbers span all values 
modulo two and three. So we can assume that the first coordinates are integers. We can also ensure that no two adjacent vertices lie on the same vertical line.

Let $P$ be the set of all prime numbers which appear in the decomposition of $\left|\phi(u)_{1}-\phi(v)_{1}\right|$ where $\phi(u)=\left(\phi(u)_{1}, \phi(u)_{2}\right), \phi(v)=\left(\phi(v)_{1}, \phi(v)_{2}\right)$ and $u v \in$ $E$. The set $P$ is finite, as no $\phi(u), \phi(v), u, v \in V$, lie on the same vertical line. Now we put horizontal lines $\mathbb{R}^{2}$ such that the distance between two consecutive lines is $\delta>0$ and every vertical line is crossed by at least $\prod_{p \in P} p$ lines in every neighborhood. Again, we declare one of these lines as initial and number them according to their order. Then, for every vertex $v \in V$, we set $\phi(v)=x$ such that $x \in \Omega(v, r)$, the first coordinate of $\phi(v)$ remains the same and $x$ lies on the horizontal line with number $l \equiv f(v)_{2}(\bmod 2), l \equiv f(v)_{2}(\bmod 3)$. In addition, if there is another prime number $p$ which divides the difference $\left|\phi(u)_{1}-\phi(v)_{1}\right|$, $u v \in E$, then we set such horizontal lines for $u$ and $v$ that their numbers are not congruent modulo $p$. The different residues modulo $p$ can be chosen according to the coloring $f$ (each color corresponds to a unique residue modulo $p>4$ ). Note that there are enough horizontal lines due to the choice of $\delta$. Then the horizontal and vertical lines form an elongated grid which we contract such that $\epsilon=\delta$. This does not violate planarity and the obtained embedding is primitive.

Acknowledgment. I would like to thank my supervisor Pavel Valtr for his time and for all the provided advice.

\section{References}

1. Balko, M.: Grid representations and the chromatic number. In: 28th European Workshop on Computational Geometry, EuroCG 2012, pp. 45-48 (2012)

2. Cáceres, J., Cortés, C., Grima, C.I., Hachimori, M., Márquez, A., Mukae, R., Nakamoto, A., Negami, S., Robles, R., Valenzuela, J.: Compact Grid Representation of Graphs. In: Márquez, A., Ramos, P., Urrutia, J. (eds.) EGC 2011. LNCS, vol. 7579, pp. 166-174. Springer, Heidelberg (2012)

3. Chappell, G.G., Gimbel, J., Hartman, C.: Thresholds for path colorings of planar graphs. Topics in Discrete Mathematics, 435-454 (2006)

4. Chrobak, M., Nakano, S.: Minimum-width grid drawings of plane graphs. Computational Geometry 11, 29-54 (1995)

5. Flores Pen̋aloza, D., Zaragoza Martinez, F.J.: Every four-colorable graph is isomorphic to a subgraph of the visibility graph of the integer lattice. In: Proceedings of the 21st Canadian Conference on Computational Geometry, CCCG 2009, pp. 91-94 (2009)

6. de Fraysseix, H., Pach, J., Pollack, R.: How to draw a planar graph on a grid. Combinatorica 10(1), 41-51 (1990)

7. Goddard, W.: Acyclic colorings of planar graphs. Discrete Math. 91, 91-94 (1991)

8. Kára, J., Pór, A., Wood, D.R.: On the chromatic number of the visibility graph of a set of points in the plane. Discrete Comput. Geom. 34, 497-506 (2005)

9. Lovász, L.: On decomposition of graphs. Studia Math. Hung. 1, 237-238 (1966)

10. Pór, A., Wood, D.R.: No-three-in-line-in-3d. Algorithmica 47, 481-488 (2007)

11. Schnyder, W.: Embedding planar graphs on the grid. In: Proceedings of the First Annual ACM-SIAM Symposium on Discrete Algorithms, SODA 1990, pp. 138-148. Society for Industrial and Applied Mathematics (1990) 